\title{
CHANGING THE DEPTH OF AN ORDERED SET BY DECOMPOSITION
}

\author{
BY
}

\author{
E. C. MILNER AND K. PRIKRY ${ }^{1}$
}

\begin{abstract}
The depth of a partially ordered set $\langle P,<\rangle$ is the smallest ordinal $\gamma$ such that $\langle P,<\rangle$ does not embed $\gamma^{*}$. The width of $\langle P,<\rangle$ is the smallest cardinal number $\mu$ such that there is no antichain of size $\mu+1$ in $P$. We show that if $\gamma>\omega$ and $\gamma$ is not an infinite successor cardinal, then any partially ordered set of depth $\gamma$ can be decomposed into $\operatorname{cf}(|\gamma|)$ parts so that the depth of each part is strictly less than $\gamma$. If $\gamma=\omega$ or if $\gamma$ is an infinite successor cardinal, then for any infinite cardinal $\lambda$ there is a linearly ordered set of depth $\gamma$ such that for any $\lambda$-decomposition one of the parts has the same depth $\gamma$. These results are used to solve an analogous problem about width. It is well known that, for any cardinal $\lambda$, there is a partial order of width $\omega$ which cannot be split into $\lambda$ parts of finite width. We prove that, for any cardinal $\lambda$ and any infinite cardinal $\nu$, there is a partial order of width $\nu^{+}$which cannot be split into $\lambda$ parts of smaller width.
\end{abstract}

1. Introduction. The depth of a partially ordered set $\langle P,<\rangle(\operatorname{depth}(\langle P,<\rangle))$ is the smallest ordinal number $\gamma$ such that $\langle P,<\rangle$ does not contain a reverse wellordered subset of length $\gamma$, i.e. there is no chain of type $\gamma^{*}$ in $\langle P,<\rangle$. For example, a well-founded partial order has depth at most $\omega$. In this paper we investigate the question whether, for given $\gamma$, there is a cardinal number $\lambda$, depending only on $\gamma$, such that any partially ordered set $(P,<)$ of depth $\gamma$ can be partitioned into $\lambda$ parts so that the depth of each part is strictly less than $\gamma$.

We show (Theorem 2.4) that if $\gamma>2, \gamma \neq \omega$, and $\gamma$ is not an infinite successor cardinal, then there is a cardinal number $\lambda=\lambda(\gamma)$ such that the following statement is true.

$S(\gamma, \lambda):$ Whenever a partially ordered set $\langle P,<\rangle$ has depth $\gamma$, there is a $\lambda$ decomposition $\left\{P_{\xi}: \xi<\lambda\right\}$ of $P$ such that $\left\langle P_{\xi},<\right\rangle$ has depth less than $\gamma$ for each $\xi<\lambda$. Clearly $S(\omega, \lambda)$ is false for every $\lambda$ (consider $\lambda^{+}$). We show (Theorem 2.5) that, if $\kappa \geq 2$ is any cardinal, $\nu$ is an infinite cardinal, and $\lambda<\max \left\{\nu^{+}, \kappa\right\}$, then for any $\lambda$-decomposition $\left\{L_{\xi}: \xi<\lambda\right\}$ of ${ }^{\nu} \kappa$ there is some $\xi<\lambda$ such that $L_{\xi}$ has the same depth $\nu^{+}$as ${ }^{\nu} \kappa$ with the ordinary lexicographic ordering. Since $\kappa$ may be chosen arbitrarily large, this shows that $S\left(\nu^{+}, \lambda\right)$ is also false for every $\lambda$.

Apart from providing a novel characterization of those ordinals which are infinite successor cardinals (Theorem 2.10), these results have further applications to the theory of partially ordered sets and also to the partition calculus. Perles has shown that the Dilworth chain decomposition theorem does not extend to partial orders in

Received by the editors February 21, 1984 and, in revised form, September 17, 1984.

1980 Mathematics Subject Classification. Primary 03E05, 04A20, 06A05, 06A10.

Key words and phrases. Partial order, depth, width, extendable ordinal, partition relation, graph, category.

${ }^{1}$ Research supported by NSERC grant \#A5198 and NSF grant \#MCS 8303601. 
which the size of antichains is not finitely bounded. More precisely, for any cardinal number $\lambda$, there is a partially ordered set which has not only finite antichains, but has also the further property that, whenever $\left\{P_{\xi}: \xi<\lambda\right\}$ is a $\lambda$-decomposition of $P$, there is some $\xi<\lambda$ such that $\left\langle P_{\xi}, \prec\right\rangle$ has an antichain of size $n$ for every $n<\omega$. We prove (Theorem 2.7) a similar result for partial orders in which the size of antichains is at most $\nu$, where $\nu$ is any infinite cardinal.

2. Statement of results. Our set-theoretic notation is fairly standard, as used, for example, in Jech [8]. Unless stated otherwise, lower case Greek letters always denote ordinal numbers. In particular, $\omega$ and $\omega_{\alpha}$ have their usual meanings and we use $\kappa, \lambda, \mu$ and $\nu$ to denote arbitrary cardinal numbers. The reverse order type of an ordinal $\alpha$ is denoted by $\alpha^{*}$. We define $\alpha^{-}$to be $\beta$ if $\alpha=\beta+1$ is a successor ordinal, and $\alpha^{-}=\alpha$ if $\alpha$ is a limit. The ordinal $\alpha$ is (additively) decomposable if it is a sum of two smaller ordinals. As usual we write ${ }^{\alpha} \beta$ for the set of all sequences of length $\alpha$ with terms in $\beta$ and we denote the lexicographic ordering of ${ }^{\alpha} \beta$ by $\prec$. The symbol $\alpha^{\beta}$ denotes ordinal exponentiation.

The partition symbol (see $[6]) \alpha \rightarrow\left(\beta_{\xi}\right)_{\xi<\lambda}^{r}$, where $r<\omega$, means the following is true: whenever $(A,<)$ is a linearly ordered set of type $\alpha$ and $\left\{C_{\xi}: \xi<\lambda\right\}$ is any $\lambda$-partition of $[A]^{r}=\{X \subseteq A:|X|=r\}$, then there are $\xi<\lambda$ and $B \subseteq A$ such that $(B,<)$ has order type $\beta_{\xi}$ and $[B]^{r} \subseteq C_{\xi}$ (i.e. $B$ is $C_{\xi}$-homogeneous).

One of the main results we prove is the following decomposition theorem for linearly ordered sets.

THEOREM 2.1. Let $\rho \geq 3, \rho \neq \omega, \rho$ is not an infinite successor cardinal and let

$$
\lambda(\rho)= \begin{cases}2 & \text { if } \rho^{-} \text {is decomposable, } \\ \operatorname{cf}(|\rho|) & \text { if } \rho^{-} \text {is indecomposable and } \rho<|\rho|^{\omega}, \\ \omega & \text { if } \rho^{-} \text {is indecomposable and } \rho \geq|\rho|^{\omega} .\end{cases}
$$

Then, for any linearly ordered set $\langle L,<\rangle$ of depth $\rho$, there is a $\lambda(\rho)$-decomposition $\left\{L_{\xi}: \xi<\lambda(\rho)\right\}$ of $L$ such that the depth of each $L_{\xi}(\xi<\lambda(\rho))$ is less than $\rho$.

In the case of Theorem 2.1 when $\rho^{-}$is indecomposable and $\rho \geq|\rho|^{\omega}$, we shall prove a stronger result (Theorem 2.2). This is really a generalization of the negative partition theorem that

$$
\begin{array}{r}
\xi \nrightarrow\left(\kappa^{n}\right)_{n<\omega}^{1} \\
\text { if } \left.\kappa \text { is an infinite cardinal and } \xi<\kappa^{+} \text {(see }[\mathbf{1 0}]\right) .
\end{array}
$$

THEOREM 2.2. If $\kappa$ is an infinite cardinal and $\langle L,<\rangle$ is a linearly ordered set of depth less than $\kappa^{+}$, then there is an $\omega$-decomposition $\left\{L_{n}: n<\omega\right\}$ of $L$ such that $L_{n}$ has depth at most $\kappa^{n}(n<\omega)$.

By repeated application of Theorem 2.1 we immediately obtain the following useful corollary.

COROLLARY 2.3. If $\langle L,<\rangle$ is a linearly ordered set of depth $\rho \geq \omega$, then there is a $\operatorname{cf}(|\rho|)$-decomposition $\left\langle L_{\xi}: \xi<\operatorname{cf}(|\rho|)\right\rangle$ of $L$ such that each $L_{\xi}$ has depth at most $|\rho|$.

A linear order type $\varphi$ is said to be extendable (see [2]), if whenever a partially ordered set $\langle P,<\rangle$ does not embed $\varphi$ (we write $\langle P,<\rangle \nsupseteq \varphi$ ), there is a linear extension $<_{1}$ of $<$ such that $\left\langle P,<_{1}\right\rangle \nsupseteq \varphi$. For example, $\eta$ the order type of the rationals 
is extendable [2]. F. Galvin and R. McKenzie (unpublished, but see [2]) have given a complete description of the class of extendable ordinals: it is the smallest class $\mathcal{U}$ such that (i) $0,1, \omega \in \mathcal{U}$, (ii) $\mathcal{U}$ is closed, and (iii) $\alpha \cdot(\operatorname{cf} \alpha)^{+} \in \mathcal{U}$ whenever $\alpha \in \mathcal{U} \backslash \omega$. Thus every infinite cardinal is extendable and so are the ordinals $\omega_{\omega} \omega_{1}, \omega_{\omega} \omega_{2}$, etc. It follows immediately from the definition that Theorem 2.1 also applies to partial orders if one adds the hypothesis that $\rho$ is an extendable ordinal. F. Galvin (unpublished) has proved a more powerful decomposition theorem for partial orders (it is related to Theorems 9 and 10 of [7]) which states: If $\nu$ is an infinite cardinal and $\langle P,<\rangle \rightarrow(\nu)_{\nu}^{1}$ (i.e. for any $\nu$-decomposition of $P$, some part contains a chain of type $\nu$ ), then $\langle P,<\rangle \geq \alpha$ for every $\alpha<\nu^{+}$. Part (i) of Theorem 2.4 is a restatement of this, and we are indebted to Galvin for pointing out that part (ii) is an easy consequence of this and Theorem 2.1.

THEOREM 2.4. Let $\nu$ be an infinite cardinal and let $\langle P,<\rangle$ be a partially ordered set having depth less than $\nu^{+}$. Then (i) there is a decomposition of $P$ into $\nu$ parts, each of depth at most $\nu$, and (ii) if $\nu$ is an uncountable limit cardinal, then there is a decomposition of $P$ into $\operatorname{cf}(\nu)$ parts, each of depth strictly less than $\nu$.

It is easily seen that $\left\langle{ }^{\nu} \kappa, \prec\right\rangle$ has depth $\nu^{+}$if $\kappa \geq 2$ and $\nu \geq \omega$. We will prove the following theorem.

THEOREM 2.5. Let $\kappa \geq 2, \nu \geq \omega, \lambda<\max \left\{\nu^{+}, \kappa\right\}$ and let $\left\langle L_{\xi}: \xi<\lambda\right\rangle$ be any $\lambda$-decomposition of ${ }^{\nu} \kappa$. Then $\left\langle L_{\xi}, \prec\right\rangle$ has depth $\nu^{+}$for some $\xi<\lambda$.

This shows that the hypothesis $\rho \neq \nu^{+}$is needed in the statement of Theorem 2.1. We remark that our original proof of this result for the case when $\nu \leq \kappa$ and $\nu$ is a singular limit cardinal was more complicated than the one presented here which uses an idea shown to us by Z. Nagy. Although more complicated, our first proof is not without interest since it provided somewhat more information about those subsets of ${ }^{\nu} \kappa$ having depth $\nu^{+}$. We shall give that proof, in a more general setting, in a later paper.

In general, the bound on $\lambda$ in Theorem 2.5 cannot be improved. This follows from the fact that we must have $\lambda<\left|{ }^{\nu} \kappa\right|$ and $\left|{ }^{\nu} \kappa\right|=\max \left\{\nu^{+}, \kappa\right\}$ if the generalized continuum hypothesis (GCH) holds and $\nu$ is not between $\operatorname{cf}(\kappa)$ and $\kappa$. The only question that remains here is whether the conclusion of Theorem 2.5 holds when $\operatorname{cf}(\kappa) \leq \nu<\kappa$ and $\lambda=\kappa$. We make the observation that this is so, provided that the partition relation

$$
\left|{ }^{\nu} \kappa\right| \rightarrow\left(\kappa^{+}, \nu\right)^{2}
$$

holds. To see this, suppose, for contradiction, that there is a $\kappa$-decomposition $\left\{L_{\xi}: \xi<\kappa\right\}$ of ${ }^{\nu} \kappa$ such that each $L_{\xi}$ has depth less than $\nu^{+}$. In view of Corollary 2.3 we may therefore suppose that each $L_{\xi}$ has depth at most $\nu$. Let ${ }^{\nu} \kappa=\left\{\chi_{\alpha}: \alpha<\right.$ $\left.\left|{ }^{\nu} \kappa\right|\right\}$ and define a function $\varphi:\left|{ }^{\nu} \kappa\right| \rightarrow \kappa$ so that $\chi_{\alpha} \in L_{\varphi(\alpha)}$. Consider the partition $\left[\left.\right|^{\nu} \kappa \mid\right]^{2}=C_{0} \cup C_{1}$, where $C_{0}=\{\{\alpha, \beta\}: \alpha<\beta<|\nu \kappa|$, either $\varphi(\alpha)<\varphi(\beta)$ or $\varphi(\alpha)=\varphi(\beta)$ and $\left.\chi_{\alpha} \prec \chi_{\beta}\right\}$. Since $\left\langle{ }^{\nu} \kappa, \prec\right\rangle \nsupseteq \kappa^{+}$, it follows that there is no $C_{0^{-}}$ homogeneous set of size $\kappa^{+}$. Therefore, by (2.2) there is a $C_{1}$-homogeneous set of size $\nu$ and this leads to the contradiction that some $L_{\xi}$ has depth greater than $\nu$.

Note that (2.2) holds when $\nu=\operatorname{cf}(\kappa)=\omega$ by the Erdős-Dushnik-Miller Theorem [4]. It also holds for $\nu=\operatorname{cf}(\kappa)>\omega$ if GCH holds (see [6, Corollary 17.8]). 
When first considering special cases of Theorem 2.5 we were naturally led to consider certain topological properties of the space ${ }^{\nu} \kappa$ (endowed with the lexicographic order topology) which generalize well-known facts about the real line. A subset of a topological space is of the first (second) $\mu$-category if it is (is not) the union of fewer than $\mu$ nowhere dense subsets. In $\S 4$ we shall prove the following result.

THEOREM 2.6. If $\nu \geq \omega, \kappa \geq 2$ and if $A_{\xi}(\xi<\nu)$ are subsets of ${ }^{\nu} \kappa$ having depth less than $\nu^{+}$, then $A=\bigcup\left\{A_{\xi}: \xi<\nu\right\}$ is of the first $(\operatorname{cf}(\nu))^{+}$-category.

We also show (Theorem 4.3) that ${ }^{\nu} \kappa$ is of the second $(\operatorname{cf}(\nu))^{+}$-category. This fact, together with Theorem 2.6, immediately gives Theorem 2.5 for the case when $\nu^{+} \geq \kappa$.

The width $\mu(\langle P,<\rangle)$ of a partially ordered set $\langle P,<\rangle$ is the smallest cardinal number $\mu$ such that $P$ does not contain an antichain of size $\mu+1$. A partially ordered set $\langle P,<\rangle$ will be said to have the property $D(\lambda)$ if and only if there is a $\lambda$-decomposition $\left\{P_{\xi}: \xi<\lambda\right\}$ of $P$ such that $\mu\left(\left\langle P_{\xi},<\right\rangle\right)<\mu(\langle P,<\rangle)$ holds for all $\xi<\lambda$. Dilworth's chain decomposition theorem $[\mathbf{3}]$ implies that any partially ordered set of finite width $k(\geq 2)$ has the property $D(2)$. Perles $[\mathbf{1 1}]$ showed that there is no sensible extension of Dilworth's theorem to partial orders of infinite width. More precisely, for any cardinal number $\lambda$, there is a partially ordered set of width $\omega$ which does not have the property $D(\lambda)$ (consider the direct product $\kappa \otimes \kappa$ where $\kappa>\lambda$ is infinite and $(\alpha, \beta) \leq\left(\alpha^{\prime}, \beta^{\prime}\right)$ if and only if $\alpha \leq \alpha^{\prime}$ and $\left.\beta \leq \beta^{\prime}\right)$. In [9] we briefly considered this problem for partial orders of uncountable width and we mentioned there (footnote, p. 463) that S. Todorčević [12] had shown that for any $\lambda$ there is a partial order of width $\omega_{1}$ not having property $D(\lambda)$. We shall now prove the following more general result.

THEOREM 2.7. If $\mu=\omega$ or $\nu^{+}$for some $\nu \geq \omega$ and if $\lambda$ is any cardinal number, then there is a partially ordered set of width $\mu$ and dimension 2 which does not have property $D(\lambda)$.

The dimension of a partial order $<$ is the smallest cardinal $\delta$ such that $<=$ $\bigcap_{\xi<\delta}<_{\xi}$ is the intersection of $\delta$ linear orders.

Note that the direct product $\kappa \otimes \kappa$ has dimension 2 , and so we need only consider the case when $\mu=\nu^{+}$is an infinite successor cardinal. Let $\ll$ be any well-ordering of ${ }^{\nu} \kappa$, where $\kappa>\lambda$ is infinite; then $<=\prec \cap \ll$ is a partial order of dimension 2 on ${ }^{\nu} \kappa$. Since $\left\langle{ }^{\nu} \kappa, \prec\right\rangle$ has depth $\nu^{+}$, it follows that any antichain of $\left\langle{ }^{\nu} \kappa,<\right\rangle$ has cardinality at most $\nu$ so that $\mu(\langle\nu \kappa,<\rangle) \leq \mu$. However, by Theorem 2.5, if $\left\langle L_{\xi}: \xi<\lambda\right\rangle$ is any $\lambda$-decomposition of ${ }^{\nu} \kappa$, then some $L_{\xi}$ has $\prec$-depth $\nu^{+}$and so the width of $\left\langle L_{\xi},<\right\rangle$ is greater than or equal to $\mu$. Thus $\left\langle{ }^{\nu} \kappa,<\right\rangle$ does not have the property $D(\lambda)$.

Theorem 2.7 does not extend to the case when $\mu$ is an uncountable limit cardinal. We shall prove the following theorem.

THEOREM 2.8. Let $\mu>\omega$ be a strong limit cardinal and let $\langle P,<\rangle$ be a partially ordered set of dimension $\delta<\operatorname{cf}(\mu)$ and width $\mu$. Then $\langle P,<\rangle$ has the property $D\left(\left|{ }^{\delta} \operatorname{cf}(\mu)\right|\right)$.

An interesting question which is left open here for the case when $\mu$ is an uncountable limit cardinal, is whether, for arbitrary $\lambda$, there is a partially ordered set of width $\mu$ not having the property $D(\lambda)$. The above theorem shows that, if $\lambda$ is a 
strong limit cardinal and $\lambda \geq \mu$, then the dimension of such a partial order must be at least $\operatorname{cf}(\mu)$.

The following result is an application of Theorem 2.7 to the theory of infinite graphs having large chromatic number without having large complete subgraphs. (Since we wrote this paper we have learned that this is a theorem of [5].)

THEOREM 2.9. Let $\mu=\omega$ or $\nu^{+}$for some infinite $\nu$ and let $\lambda$ be any cardinal. Then there is a graph $G=(V, E)$ which does not contain a complete subgraph of size $\mu$ and is such that, for any $\lambda$-decomposition $\left\{V_{\xi}: \xi<\lambda\right\}$ of the vertex set $V$, there is some $\xi<\lambda$ such that $V_{\xi}$ contains a complete subgraph of size $\mu^{\prime}$ for any $\mu^{\prime}<\mu$.

To see this simply consider the complement of the comparability graph ( $x$ and $y$ are joined by an edge if and only if $x \nless y$ and $y \nless x$ ) of a partial order $\langle P,<\rangle$ of width $\mu$ and not having property $D(\lambda)$. We do not know if Theorem 2.9 is true when $\mu$ is an uncountable limit cardinal. (This is Problem 1 of [5].)

We conclude by noting that Theorems 2.1 and 2.5 provide the following characterizations for the class of ordinal numbers comprising $\omega$ and the infinite successor cardinals.

THEOREM 2.10. Let $\rho \geq 3$ and let $\lambda(\rho)$ be given by (2.1). Then the following statements are equivalent.

(i) $\rho=\omega$ or $\rho=\nu^{+}$is an infinite successor cardinal;

(ii) for every cardinal $\lambda$ there is a linearly ordered set of depth $\rho$ which is preserved under arbitrary $\lambda$-decompositions;

(iii) there is a linearly ordered set of depth $\rho$ and some $\lambda \geq \lambda(\rho)$ such that the depth is preserved under arbitrary $\lambda$-decompositions.

REMARK. In (ii) and (iii) we may replace "linearly" by "partially" thereby obtaining further equivalences.

3. Proofs of Theorems 2.1, 2.2 and 2.4. Lemmas 3.1-3.3 deal with special cases of Theorem 2.1 and provide the inductive step needed for the proof of Theorem 2.2. Lemma 3.1 is stated for arbitrary partial orders. We use the notation $P(>a)$ to denote the set of all elements $x$ of the partially ordered set $\langle P,<\rangle$ such that $x>a$.

LEMMA 3.1. If the partially ordered set $\langle P,<\rangle$ has depth $\rho$ and if $\rho^{-}$is decomposable, then there is a partition $P=P_{0} \cup P_{1}$ such that each $\left\langle P_{\xi},<\right\rangle(\xi<2)$ has depth less than $\rho$.

Proof. Since $\rho^{-}$is decomposable, we may write $\rho=\alpha+\beta$, where $\rho>\beta>1$. Let $P_{0}=\left\{x \in P: P(>x) \geq \alpha^{*}\right\}, P_{1}=P \backslash P_{0}$. Then $P_{0}$ has depth at most $\beta$ and $P_{1}$ has depth at most $\alpha+1$.

LEMMA 3.2. If the linearly ordered set $\langle L,<\rangle$ has depth $\rho=\beta+1$, where $\beta$ is a limit ordinal, then $L=\bigcup\{I(a): a \in A\}$, where $A$ is a subset of $L$ with order type $(\operatorname{cf}(\beta))^{*}$ and the $I(a)(a \in A)$ are pairwise disjoint subintervals of $L$ such that $a \in I(a)$ and $I(a)$ has depth less than $\rho$.

ProOF. Since the depth of $L$ is $\rho$, it contains a coinitial subset of type $\beta^{*}$ and hence there is a coinitial subset $A=\left\{a_{\xi}: \xi<\operatorname{cf}(\beta)\right\}$ of $L$ with $a_{0}>a_{1}>a_{2}>\cdots$. 
Define the intervals $I\left(a_{\xi}\right)(\xi<\operatorname{cf}(\beta))$ inductively so that

$$
I\left(a_{\xi}\right)=L\left(>a_{\xi+1}\right) \backslash \bigcup\left\{I\left(a_{\eta}\right): \eta<\xi\right\} .
$$

LEMMA 3.3. If the linearly ordered set $\langle L,<\rangle$ has depth $\rho$ and if $\rho$ is an infinite limit ordinal, then $L=\{I(a): a \in A\}$, where $A$ is a subset of $L$ having depth at most $\operatorname{cf}(\rho)$ and the $I(a)(a \in A)$ are pairwise disjoint subintervals of $L$ such that $a \in I(a)$ and $I(a)$ has depth less than $\rho$.

Proof. Without loss of generality we may assume that $L$ is complete so that every nonempty subinterval of $L$ has a leii-hand and a right-hand endpoint. Let $\left\langle\rho_{\xi}: \xi<\operatorname{cf}(\rho)\right\rangle$ be a strictly increasing sequence of ordinals cofinal in $\rho$.

Let $M=\{x \in L:(x, y)$ has depth $\rho$ for all $y>x\}$ and let $\bar{M}$ be the closure of $M$ in $L$. Note that, if $x \in M$, then $L(>x)$ has coinitiality strictly less than $\operatorname{cf}(\rho)$. Otherwise there are points $y_{\xi} \in L(>x)(\xi<\operatorname{cf}(\rho))$ such that $y_{0}>y_{1}>\cdots$ and the interval $\left[y_{\xi+1}, y_{\xi}\right]$ contains a subset of order type $1+\rho_{\xi}^{*}$, which implies that $\langle L,<\rangle \geq \rho^{*}$. For a similar reason it follows that $M$, and hence $\bar{M}$, has depth at $\operatorname{most} \operatorname{cf}(\rho)$.

Consider a maximal interval $I$ of $L$ disjoint from $\bar{M}$ and suppose the left-hand endpoint of $I$, say $x$, belongs to $M$. Since $L(>x)$ has coinitiality strictly less than $\operatorname{cf}(\rho)$ we can partition $I$ into fewer than $\operatorname{cf}(\rho)$ disjoint subintervals whose left-hand endpoints do not belong to $M$ (they are points of $I$ ). It follows from this that $L \backslash \bar{M}$ is a union of disjoint intervals $\left\{I(a): a \in A_{1}\right\}$, where $A_{1}$ is a subset of $L$ having depth at most $\operatorname{cf}(\rho)$ and $I(a)\left(a \in A_{1}\right)$ is an interval which contains $a$ and whose left-hand endpoint does not belong to $M$.

Consider an interval $I(a)\left(a \in A_{1}\right)$ having left-hand and right-hand endpoints $x, y$. Then there is some ordinal $\tau$ and a strictly increasing closed sequence $\left\langle x_{\sigma}: \sigma \leq\right.$ $\tau)$ in $L$ such that $x_{0}=x, x_{\tau}=y$ and the interval $\left[x_{\sigma}, x_{\sigma+1}\right]$ has depth less than $\rho$ for all $\sigma<\tau$. This is easy to see, because if $x_{\sigma}<y$ has already been chosen, then, since $x_{\sigma} \notin M$, there is some $x_{\sigma+1}\left(x_{\sigma}<x_{\sigma+1} \leq y\right)$ such that $\left[x_{\sigma}, x_{\sigma+1}\right]$ has depth less than $\rho$, and the construction must terminate for some $\tau$ with $x_{\tau}=y$. Replacing each $I(a)\left(a \in A_{1}\right)$ by such a well-ordered sequence of (disjoint) subintervals, we see that $L \backslash \bar{M}=\bigcup\left\{I(a): a \in A_{2}\right\}$, where the interval $I(a)\left(a \in A_{2}\right)$ contains $a$ and has depth less than $\rho$ and $A_{2}$ has depth at most $\operatorname{cf}(\rho)$. The lemma follows if we set $A=A_{2} \cup \bar{M}$ and put $I(a)=\{a\}$ for $a \in \bar{M}$.

PROOF OF THEOREM 2.2. For $\rho<\kappa^{\omega}$ the result is obvious and so we assume that $\kappa^{\omega} \leq \rho<\kappa^{+}$and use induction on $\rho$. Suppose $\langle L,<\rangle$ has depth $\rho$. Since $\operatorname{cf}(\rho)$ and $\operatorname{cf}\left(\rho^{-}\right)$are less than or equal to $\kappa$, it follows from one of Lemmas 3.1, 3.2, or 3.3 that $L=\bigcup\{I(a): a \in A\}$, where $A$ is a subset of $L$ having depth at most $\kappa+1$ and the $I(a)(a \in A)$ are pairwise disjoint subintervals of $L$ such that $a \in I(a)$ and $I(a)$ has depth less than $\rho$. By the induction hypothesis, each $I(a)(a \in A)$ is a union of $\omega$ sets $X_{n}(a)(n<\omega)$ such that $X_{n}(a)$ has depth at most $\kappa^{n}$. Put $L_{0}=L_{1}=\varnothing, L_{n+2}=\bigcup\left\{X_{n}(a): a \in A\right\}(n<\omega)$. Then $L=\bigcup\left\{L_{n}: n<\omega\right\}$ and the result follows since the depth of $L_{n+2}$ is at most $\kappa^{n+1}+1$ (this follows from the fact that if $\alpha_{\rho}<\kappa^{n}(\rho<\kappa)$, then $\left.\sum_{\rho<\kappa} \alpha_{\rho} \leq \kappa^{n+1}\right)$.

As an easy corollary to Lemma 3.3 we have the following result.

LEMMA 3.4. If $\rho$ is a singular cardinal and if $\langle L,<\rangle$ is a linearly ordered set of depth $\rho$, then $L$ is a union of $\operatorname{cf}(\rho)$ sets $L_{\sigma}(\sigma<\operatorname{cf}(\rho))$ each of depth less than $\rho$. 
ProOF. Let $\left\langle\rho_{\sigma}: \sigma<\operatorname{cf}(\rho)\right\rangle$ be a sequence of cardinals cofinal in $\rho$. By Lemma 3.3, $L=\bigcup\{I(a): a \in A\}$, where $A$ is a subset of $L$ of $\operatorname{depth}$ at most $\operatorname{cf}(\rho)$ and the $I(a)(a \in A)$ are pairwise disjoint intervals of depth strictly less than $\rho$ such that $a \in I(a)$. Put

$$
L_{\sigma}=\bigcup\left\{I(a): a \in A \text { and the depth of } I(a) \text { is } \leq \rho_{\sigma}\right\} \quad(\sigma<\operatorname{cf}(\rho)) .
$$

Clearly $L=\bigcup\left\{L_{\sigma}: \sigma<\operatorname{cf}(\rho)\right\}$. Also, if $X$ is a reverse well-ordered subset of $L_{\sigma}$, then $X=\bigcup\left\{X(a): a \in A_{1}\right\}$, where $A_{1}$ is a reverse well-ordered subset of $A$ and $X(a)\left(a \in A_{1}\right)$ is a reverse well-ordered subset of $I(a)$. Therefore $\left|A_{1}\right|<\operatorname{cf}(\rho)$ and $|X(a)|<\rho_{\sigma}\left(a \in A_{1}\right)$ and so $|X| \leq \rho_{\sigma} \cdot \operatorname{cf}(\rho)$. Thus, $L_{\sigma}(\sigma<\operatorname{cf}(\rho))$ has depth at most $\left(\rho_{\sigma} \cdot \operatorname{cf}(\rho)\right)^{+}$, which is less than $\rho$.

The next lemma is a weak version of Corollary 2.3. The result is also true for partially ordered sets by an unpublished theorem of F. Galvin (see Proof of Theorem 2.4). In the special case $\kappa=\omega$, Baumgartner and Hajnal [1] proved the much stronger result that, for any linear order $\varphi, \varphi \rightarrow(\omega)_{\omega}^{1}$ implies $\varphi \rightarrow(\alpha)_{n}^{2}$ for any $\alpha<\omega_{1}$ and $n<\omega$; this result has been extended to arbitrary partial order types $\varphi$ by S. Todorčević $[\mathbf{1 3}]$.

LEMMA 3.5. If $\kappa$ is an infinite cardinal and $\langle L,<\rangle$ is a linearly ordered set of depth $\rho<\kappa^{+}$, then $L$ is a union of $\kappa$ sets $L_{\xi}(\xi<\kappa)$ each having depth at most $\kappa$.

PROOF. For $\rho=\kappa$ the result is obvious; just put $L_{\xi}=L(\xi<\kappa)$. Now assume that $\rho>\kappa$ and use induction on $\rho$.

If $\rho^{-}$is decomposable or if $\rho=\beta+1$ and $\beta$ is a limit, then the result follows immediately from Lemma 3.1 or 3.2 and the induction hypothesis.

Suppose $\rho$ is a limit ordinal. Then by Lemma 3.3, $L=\bigcup\{I(a): a \in A\}$, where $A$ is a subset of $L$ having depth at most $\operatorname{cf}(\rho), a \in I(a)$, and the $I(a)(a \in A)$ are disjoint subintervals of $L$ each having depth less than $\rho$. By the induction hypothesis we may write $I(a)=\bigcup\{X(a, \xi): \xi<\kappa\}(a \in A)$, and each $X(a, \xi)(a \in A, \xi<\kappa)$ has depth at most $\kappa$.

Case $1 . \kappa$ is regular.

Put $L_{\xi}=\bigcup\{X(a, \xi): a \in A\}(\xi<\kappa)$. Clearly, $L=\bigcup\left\{L_{\xi}: \xi<\kappa\right\}$. Also, if $X$ is a reverse well-ordered subset of $L_{\xi}$, then $X=\bigcup\left\{X^{\prime}(a, \xi): a \in A_{1}\right\}$, where $A_{1}$ is a reverse well-ordered subset of $A$ and $X^{\prime}(a, \xi)\left(a \in A_{1}\right)$ is a reverse well-ordered subset of $X(a, \xi)$. Therefore, $\left|A_{1}\right|<\operatorname{cf}(\rho) \leq \kappa$ and $\left|X^{\prime}(a, \xi)\right|<\kappa\left(a \in A_{1}\right)$, and so $|X|<\kappa$. Thus, the depth of $L_{\xi}(\xi<\kappa)$ is at most $\kappa$.

Case 2. $\kappa$ is singular.

Since $X(a, \xi)(a \in A, \xi<\kappa)$ has depth at most $\kappa$, it follows from Lemma 3.4 that $X(a, \xi)=\bigcup\{X(a, \xi, \sigma): \sigma<\operatorname{cf}(\kappa)\}$ and the sets $X(a, \xi, \sigma)(\sigma<\operatorname{cf}(\kappa))$ have depth strictly less than $\kappa$. Let $\left\langle\kappa_{\eta}: \eta<\operatorname{cf}(\kappa)\right\rangle$ be a sequence of cardinals cofinal in $\kappa$ and, for $\eta<\operatorname{cf}(\kappa), \xi<\kappa$, put

$$
L_{\eta}(\xi)=\bigcup\left\{X(a, \xi, \sigma): a \in A, \sigma<\operatorname{cf}(\kappa) \text { and the depth of } X(a, \xi, \sigma) \text { is } \leq \kappa_{\eta}\right\} .
$$

Then $L$ is the union of the sets $L_{\eta}(\xi)$. It remains only to show that $L_{\eta}(\xi)(\eta<$ $\operatorname{cf}(\kappa), \xi<\kappa)$ has depth at most $\kappa$. Suppose $X$ is a reverse well-ordered subset of $L_{\eta}(\xi)$. Then

$$
X=\bigcup\left\{X^{\prime}(a, \xi, \sigma): a \in A_{1}, \sigma<\operatorname{cf}(\kappa)\right\}
$$


where $A_{1}$ is a reverse well-ordered subset of $A$ and $X^{\prime}(a, \xi, \sigma)$ is a reverse wellordered subset of $X(a, \xi, \sigma)$ of cardinality less than $\kappa_{\eta}$. Since $\left|A_{1}\right|<\operatorname{cf}(\rho)<\kappa$, it follows that $|X|<\kappa$ and so the depth of $L_{\eta}(\xi)$ is at most $\kappa$.

For the proof of Theorem 2.1 we need the following lemma.

LEMMA 3.6. Let $\mu$ be an infinite cardinal number. If $\left\langle s_{\sigma}: \sigma<\mu^{+}\right\rangle$is a descending sequence of type $\left(\mu^{+}\right)^{*}$ in ${ }^{\theta} 2$, then $\left|\left\{\varphi<\theta: s_{\sigma}(\varphi)=0\right\}\right| \geq \mu$ for some $\sigma<\mu^{+}$.

Proof. For distinct $s, s^{\prime} \in{ }^{\theta} 2$ let $\delta\left(s, s^{\prime}\right)$ denote the least ordinal $\delta<\theta$ such that $s(\delta) \neq s^{\prime}(\delta)$. Clearly, if $s^{\prime \prime} \prec s^{\prime} \prec s$, then $\delta\left(s, s^{\prime \prime}\right) \leq \delta\left(s, s^{\prime}\right)$. It follows from this that there is a sequence of ordinals $\left\langle\sigma(\eta): \eta\left\langle\mu^{+}\right\rangle\right.$in $\mu^{+}$such that $0=\sigma(0)<\sigma(1)<\cdots$ and $\delta\left(s_{\sigma(\eta)}, s_{\sigma}\right)=\delta_{\eta}$ for $\eta<\mu^{+}$and $\sigma(\eta+1) \leq \sigma<\mu^{+}$. Clearly $\delta_{0}<\delta_{1}<\cdots$ and $s_{\sigma(\mu)}\left(\delta_{\eta}\right)=0$ for all $\eta<\mu$.

Proof OF THEOREM 2.1. Assume that $\rho \neq \omega, \rho \geq 3$ is not an infinite successor cardinal, and that $\lambda(\rho)$ is given by $(2.1)$. Let $\langle L,<\rangle$ be a linearly ordered set of depth $\rho$. We have to show that $L$ is a union of $\lambda(\rho)$ sets each of depth less than $\rho$.

If $\rho^{-}$is decomposable, this is just Lemma 3.1. If $\rho^{-}$is indecomposable and $\rho \geq|\rho|^{\omega}$, the result follows from Theorem 2.2. Also, if $\rho>|\rho|$ and $|\rho|$ is regular, the result follows from Lemma 3.5. Therefore, we need only consider the following two cases (which overlap in the case when $\rho$ is a singular cardinal): Case 1. $\rho^{-}$is indecomposable, $\rho<|\rho|^{\omega}$ and $|\rho|$ is a singular cardinal. Case 2. $\rho$ is an uncountable limit cardinal.

Case 1. By Lemma 3.5, $L$ is a union of $|\rho|$ sets $L_{\xi}(\xi<|\rho|)$ each having depth at most $|\rho|$. By Lemma 3.4, each $L_{\xi}(\xi<|\rho|)$ is a union of $\operatorname{cf}(|\rho|)$ sets $L_{\xi \sigma}(\sigma<\operatorname{cf}(|\rho|))$ having depths less than $|\rho|$. Let $\left\langle\lambda_{\eta}: \eta<\operatorname{cf}(|\rho|)\right\rangle$ be a sequence of cardinals cofinal in $|\rho|$. For $\eta<\operatorname{cf}(|\rho|)$, let

$$
M_{\eta}=\bigcup\left\{L_{\xi \sigma}: \xi<\lambda_{\eta}, \sigma<\operatorname{cf}(|\rho|) \text { and the depth of } L_{\xi \sigma} \text { is at most } \lambda_{\eta}\right\} .
$$

Then $L$ is the union of the sets $M_{\eta}(\eta<\operatorname{cf}(|\rho|))$ and the depth of $M_{\eta}$ is at most $\left(\lambda_{\eta} \cdot \operatorname{cf}(|\rho|)\right)^{+}$which is less than $|\rho|$.

Case 2. $\rho$ an uncountable limit cardinal.

Let $\lambda=\operatorname{cf}(\rho)$ and let $\left\langle\rho_{\xi}: \xi<\lambda\right\rangle$ be a strictly increasing sequence of cardinals with limit $\rho$.

By repeated bisection it is easily seen that there is some ordinal $\theta$ and a mapping $\Psi$ from $\bigcup_{\varphi \leq \theta}{ }^{\varphi} 2$ into the set of subintervals of $\langle L,<\rangle$ so that the following conditions are satisfied:

(i) $L=\bigcup\{\Psi(s): s \in \varphi 2\}(\varphi \leq \theta)$;

(ii) $\Psi(s)$ precedes $\Psi\left(s^{\prime}\right)$ in the ordering of $L$ if $s, s^{\prime} \in \varphi^{\varphi}, \varphi \leq \theta$ and $s \prec s^{\prime}$;

(iii) if $s \in \varphi_{2}$ and $\varphi<\theta$, then $\Psi(s)=\Psi\left(s_{0}\right) \cup \Psi\left(s_{1}\right)$, where $s_{\varepsilon} \in{ }^{\varphi+1} 2, s_{\varepsilon} \mid \varphi=s$, $s_{\varepsilon}(\varphi)=\varepsilon(\varepsilon=0,1)$, and if $\Psi(s) \neq \varnothing$, then $\Psi\left(s_{1}\right)$ has a first element $x(s)$ and, moreover, if $|\Psi(s)|>1$, then $\Psi\left(s_{0}\right)$ is also nonempty;

(iv) if $\varphi \leq \theta$ is a limit ordinal and $s \in \varphi^{\varphi}$, then $\Psi(s)=\bigcap_{\varphi^{\prime}<\varphi} \Psi\left(s \mid \varphi^{\prime}\right)$;

(v) $|\Psi(s)| \leq 1$ for all $s \in{ }^{\theta} 2$.

We claim that, if $s \in{ }^{\theta} 2$ and $\Psi(s) \neq \varnothing$, then $N(s)=\{\varphi<\theta: s(\varphi)=0\}$ has cardinality $|N(s)|<\rho$. To see this, suppose, for contradiction, that there are $\varphi_{\eta} \in N(s)(\eta<\rho)$ such that $\varphi_{0}<\varphi_{1}<\cdots$. Since $\Psi\left(s \mid \varphi_{\eta}+1\right) \supseteq \Psi(s) \neq \varnothing$, 
it follows that $\Psi\left(s \uparrow \varphi_{\eta}+1\right)$ precedes $x_{\eta}=x\left(s \uparrow \varphi_{\eta}\right)$ in the ordering of $L$. Also, since $x_{\eta^{\prime}} \in \Psi\left(s \mid \varphi_{\eta^{\prime}}\right) \subseteq \Psi\left(s \uparrow \varphi_{\eta}+1\right)$, it follows that $x_{\eta^{\prime}}<x_{\eta}$ in $L$ if $\eta<\eta^{\prime}<\rho$. This contradicts the hypothesis that $L$ has depth $\rho$.

Now consider the $\lambda$-decomposition $\left\{L_{\xi}: \xi<\lambda\right\}$ of $L$, where

$$
L_{\xi}=\bigcup\left\{\Psi(s): s \in{ }^{\theta} 2,|N(s)|<\rho_{\xi}\right\} \quad(\xi<\lambda) .
$$

It follows from Lemma 3.6 that $L_{\xi}$ has depth at most $\rho_{\xi}^{+}<\rho$ for $\xi<\lambda$ and the proof of Theorem 2.1 is complete.

PROOF OF THEOREM 2.4(ii). Suppose the partially ordered set $\langle P,<\rangle$ has depth less than $\nu^{+}$, where $\nu$ is an uncountable limit cardinal. We distinguish two cases. Case 1. $\nu$ is regular. By Galvin's theorem (Theorem 2.4(i)), there is a decomposition of $P$ into $\nu$ parts each having depth at most $\nu$. By Theorem 2.1 and the fact that $\nu$ is extendable, each of these parts may be further decomposed into $\nu$ subsets each having depth strictly less than $\nu$. Case 2. $\nu$ is singular. In this case $\nu^{+}$is a limit of extendable ordinals, and so there is a linear extension $<_{1}$ of $<$ such that $\left\langle P,<_{1}\right\rangle$ has depth less than $\nu^{+}$. By Corollary 2.3 and one further application of Theorem 2.1, we see that $P$ may be decomposed into $\operatorname{cf}(\nu)$ parts such that each part has $<_{1}$-depth, and hence $<$-depth, strictly less than $\nu$.

4. Topological properties of $\nu \kappa$. Throughout this section we assume that $\nu \geq \omega$ and $\kappa \geq 2$. As in $\S 3$ for distinct elements $f, g \in{ }^{\nu} \kappa$ we denote by $\delta(f, g)$ the least ordinal $\delta$ such that $f(\delta) \neq g(\delta)$. We also write $f * g$ to denote the concatenation of any two sequences $f$ and $g$.

In this section we establish the category properties mentioned in $\S 2$ for the space ${ }^{\nu} \kappa$ endowed with the lexicographic order topology. As usual, if $X$ and $Y$ are subsets of a topological space, then $X$ is dense in $Y$ if $X \cap Y \cap U \neq \varnothing$ for every open set $U$ such that $Y \cap U \neq \varnothing . X$ is nowhere dense if it is not dense in any open set $U \neq \varnothing$. As stated previously (in $\S 2$ ), a set is of the first (second) $\mu$-category if it is (is not) the union of fewer than $\mu$ nowhere dense sets. It is easily seen that a space is of the second $\mu$-category if it has the $\mu$-Baire property, i.e. the intersection of fewer than $\mu$ dense open sets is dense.

In Theorem 4.3 below we show that ${ }^{\nu} \kappa$ with the $\prec$-order topology is $(\operatorname{cf}(\nu))^{+}$Baire and hence of the second $(\operatorname{cf}(\nu))^{+}$-category. Our proof of this is similar to the standard proof that the real line is $\omega_{1}$-Baire, but there is a difference. In the case of the real line one makes use of the fact that if $\left(a_{n}, b_{n}\right)(n<\omega)$ is a strictly decreasing nest of open intervals (i.e. $a_{0}<a_{1}<\cdots<b_{1}<b_{0}$ ), then the intersection $\bigcap_{n<\omega}\left(a_{n}, b_{n}\right)$ is nonempty. The corresponding statement is not true in ${ }^{\nu} \kappa$ (e.g. consider $a_{n}, b_{n} \in \omega^{\omega_{1}} \omega(n<\omega)$, where $a_{n}(\rho)=0(\rho \neq \omega), a_{n}(\omega)=n$, $\left.b_{n}(\rho)=0(\rho \neq n), b_{n}(n)=1\right)$. To avoid this difficulty we consider intervals $(a, b)$ of ${ }^{\nu} \kappa$ of a special kind. We call $(a, b)$ an $\varepsilon$-interval if $a \neq b$ and $a(\rho)=1-b(\rho)=0$ for all $\rho$ such that $\delta(a, b) \leq \rho<\nu$.

LEMMA 4.1. Let I be a nonempty open interval of ${ }^{\nu} \kappa$. Then there is an $\varepsilon$ interval $(a, b) \subseteq I$.

Proof. Choose $f, g, h \in I$ such that $f \prec g \prec h$. Let $\delta=\max \{\delta(f, g), \delta(g, h)\}$ and set $a=\left(g\lceil\delta+1) * 0_{\nu}\right.$ and $b=\left(g\lceil\delta+1) * 1_{\nu}\right.$, where $0_{\nu}$ and $1_{\nu}$, respectively, denote the constant sequences of ${ }^{\nu} 2$ with all terms 0 and 1 . 
LEMMA 4.2. Let $\left(f_{\alpha}, g_{\alpha}\right)(\alpha<\theta)$ be a strictly decreasing nest of $\varepsilon$-intervals in ${ }^{\nu} \kappa$ i.e. $\left(f_{\alpha} \prec f_{\alpha^{\prime}} \prec g_{\alpha^{\prime}} \prec g_{\alpha}\right.$ for $\left.\alpha<\alpha^{\prime}<\theta\right)$. Then $\theta \leq \nu$ and $\bigcap_{\alpha<\theta}\left(f_{\alpha}, g_{\alpha}\right) \neq \varnothing$. Moreover, if $\operatorname{cf}(\theta) \neq \operatorname{cf}(\nu)$, then $\bigcap_{\alpha<\theta}\left(f_{\alpha}, g_{\alpha}\right)$ contains an $\varepsilon$-interval.

Proof. Let $\delta_{\alpha}=\delta\left(f_{\alpha}, g_{\alpha}\right)(\alpha<\theta)$. Then $\delta_{0}<\delta_{1}<\cdots<\delta_{\alpha}<\cdots<\nu(\alpha<$ $\theta$ ). Therefore $\theta \leq \nu$. Let $\delta=\sup \left\{\delta_{\alpha}: \alpha<\theta\right\}$ and let $f, g \in{ }^{\nu} \kappa$ be defined by $f(\rho)=g(\rho)=\sup \left\{f_{\alpha}(\rho): \alpha<\theta\right\}$ for $\rho<\delta$ (note that this supremum is attained), and $f(\rho)=1-g(\rho)=0$ for $\delta \leq \rho<\nu$. Thus $f, g \in \bigcap_{\alpha<\theta}\left(f_{\alpha}, g_{\alpha}\right) \neq \varnothing$. Also, if $\operatorname{cf}(\theta) \neq \operatorname{cf}(\nu)$, then $\delta<\nu$ and $(f, g)$ is an $\varepsilon$-interval.

THEOREM 4.3. ${ }^{\nu} \kappa$ is $(\operatorname{cf}(\nu))^{+}$-Baire.

PROOF. Let $I_{\alpha}(\alpha<\operatorname{cf}(\nu))$ be dense open subsets of ${ }^{\nu} \kappa$. We want to show that $\bigcap_{\alpha<\operatorname{cf}(\nu)} I_{\alpha} \cap I \neq \varnothing$ for any nonempty open interval $I$. By Lemmas 4.1 and 4.2 we can inductively choose a strictly decreasing nest of $\varepsilon$-intervals $\left(a_{\alpha}, b_{\alpha}\right) \subseteq$ $\bigcap_{\beta \leq \alpha} I_{\beta} \cap I(\alpha<\operatorname{cf}(\nu))$. Then

$$
\bigcap_{\alpha<\operatorname{cf}(\nu)} I_{\alpha} \cap I \supseteq \bigcap_{\alpha<\operatorname{cf}(\nu)}\left(a_{\alpha}, b_{\alpha}\right) \neq \varnothing .
$$

In order to prove Theorem 2.6 we first note the following easy lemma.

LEMMA 4.4. If $A$ is dense in some proper interval $I$ of ${ }^{\nu} \kappa$, then $\langle A, \prec\rangle$ embeds $\nu^{*}$ and $\nu$.

Proof. Choose $f, g, h \in I$ so that $f \prec g \prec h$ and let $\delta=\max \{\delta(f, g), \delta(g, h)\}$. Let $e_{\alpha}, e_{\alpha}^{\prime}(\alpha<\nu)$ be the sequences in $\nu_{2}$ defined by setting $e_{\alpha}(\rho)=0$ for all $\rho \neq 1+\alpha, e_{\alpha}(1+\alpha)=1, e_{\alpha}^{\prime}(\rho)=1-e_{\alpha}(\rho)(\rho<\nu)$. Put $f_{\alpha}=(g \uparrow \delta+1) * e_{\alpha}$ and $h_{\alpha}=\left(g\lceil\delta+1) * e_{\alpha}^{\prime}(\alpha<\nu)\right.$. Then $f \prec f_{\alpha^{\prime}} \prec f_{\alpha} \prec h_{\alpha} \prec h_{\alpha^{\prime}} \prec h$ for $\alpha<\alpha^{\prime}<\nu$. Since $A$ is dense in $I$ and each of the intervals $\left(f_{\alpha+1}, f_{\alpha}\right),\left(h_{\alpha}, h_{\alpha+1}\right)(\alpha<\nu)$ is nonempty, it follows that $\langle A, \prec\rangle$ embeds both $\nu$ and $\nu^{*}$.

PROOF OF THEOREM 2.6. By Corollary 2.3 we can assume without loss of generality that $\operatorname{depth}\left(A_{\xi}\right) \leq \nu$ for all $\xi<\nu$, and so $A_{\xi}$ is nowhere dense by Lemma 4.4. (We remark that Corollary 2.3 is not really needed here since an easy induction, using Lemma 4.4, shows that if $A$ is somewhere dense in ${ }^{\nu} \kappa$, then $\langle A, \prec\rangle$ embeds $\alpha$ and $\alpha^{*}$ for any $\alpha<\nu^{+}$.)

If $\nu$ is regular, then we are done since $A=\bigcup_{\xi<\nu} A_{\xi}=\bigcup_{\xi<\operatorname{cf}(\nu)} A_{\xi}$ is clearly of the first $(\operatorname{cf}(\nu))^{+}$-category. We therefore assume that $\nu$ is singular and that $\left\langle\nu_{\alpha}: \alpha<\operatorname{cf}(\nu)\right\rangle$ is a strictly increasing sequence of cardinal numbers with limit $\nu$.

It is enough to prove the theorem when we have only some $\lambda<\nu$ sets $A_{\xi}(\xi<\lambda)$. For, if the theorem holds for this case, then $A_{\alpha}^{\prime}=\bigcup\left\{A_{\xi}: \xi<\nu_{\alpha}\right\}(\alpha<\operatorname{cf}(\nu))$ is of the first $(\operatorname{cf}(\nu))^{+}$-category and hence so is $A=\bigcup\left\{A_{\alpha}^{\prime}: \alpha<\operatorname{cf}(\nu)\right\}$ also.

So assume we have some $\lambda(<\nu)$ sets $A_{\xi}(\xi<\lambda)$ such that $\operatorname{depth}\left(A_{\xi}\right) \leq \nu$. We can assume $\operatorname{cf}(\nu) \leq \lambda<\nu$. Therefore, by the decomposition theorem (Theorem 2.1) (or by Lemma 3.4), we can assume further that, for each $\xi<\lambda$, there is some $\alpha(\xi)<\operatorname{cf}(\nu)$ such that $\operatorname{depth}\left(A_{\xi}\right) \leq \nu_{\alpha(\xi)}$. For $\alpha<\operatorname{cf}(\nu)$ let

$$
B_{\alpha}=\bigcup\left\{A_{\xi}: \alpha(\xi) \leq \alpha\right\} \text {. }
$$

Clearly, $\operatorname{depth}\left(B_{\xi}\right) \leq\left(\nu_{\alpha} \cdot \lambda\right)^{+}<\nu$, and so $B_{\alpha}$ is nowhere dense by Lemma 4.4. It follows that

is of the first $(\operatorname{cf}(\nu))^{+}$-category.

$$
A=\bigcup\left\{A_{\xi}: \xi<\lambda\right\}=\bigcup\left\{B_{\alpha}: \alpha<\operatorname{cf}(\nu)\right\}
$$


We conclude this section with the following simple result. We write ${ }^{<\nu} \kappa=$ $\bigcup_{\alpha<\nu}{ }^{\alpha} \kappa$.

THEOREM 4.5. If $\kappa \geq 2$ and $\nu$ is a regular infinite cardinal, and if $A \subseteq{ }^{\nu} \kappa$ has cardinality $|A|>\left.\right|^{<\nu} \kappa \mid$, then $\langle A, \prec\rangle$ has depth $\nu^{+}$.

PROOF. Suppose, for contradiction, that $\langle A, \prec\rangle$ has depth $\alpha<\nu^{+}$. Then, by Corollary 2.3, there is a $\nu$-decomposition $\left\{A_{\xi}: \xi<\nu\right\}$ of $A$ such that each $A_{\xi}$ has depth at most $\nu$. Since some $A_{\xi}$ also has cardinality $\left|A_{\xi}\right|>|<\nu \kappa|$, we may assume that $\langle A, \prec\rangle$ has depth $\leq \nu$. We will obtain a contradiction by showing that $\langle A, \prec\rangle \geq \nu^{*}$.

We claim first that there is some $f \in A$ such that, for every $\gamma<\nu$ there is $f_{\gamma} \in A$ such that $\delta\left(f, f_{\gamma}\right) \geq \gamma$ and $f \prec f_{\gamma}$. Suppose the claim is false. Then for each $f \in A$ there is $\gamma(f)<\nu$ such that $g \prec f$ for all $g \in A \backslash\{f\}$ such that $\delta(f, g) \geq \gamma(f)$. There is a subset $A^{\prime} \subseteq A$ of cardinality $\left|A^{\prime}\right|>\left.\right|^{<\nu} \kappa \mid$ such that $\gamma(f)=\gamma$ for all $f \in A^{\prime}$. Since $\left|A^{\prime}\right|>\left|{ }^{\nu} \kappa\right|$, there are distinct $f, g \in A^{\prime}$ such that $f \uparrow \gamma=g \uparrow \gamma$, and since either $f \prec g$ or $g \prec f$ we contradict the fact that $\gamma(f)=\gamma(g)=\gamma$. This proves the claim.

For each $\gamma<\nu$ choose $f_{\gamma} \in A$ such that $f_{\gamma} \prec f$ and $\delta_{\gamma}=\delta\left(f_{\gamma}, f\right) \geq \gamma$. Since $\nu$ is regular, we may assume that $\left\langle\delta_{\gamma}: \gamma<\nu\right\rangle$ is strictly increasing and this implies that $f_{0} \succ f_{1} \succ f_{2} \succ \cdots$. This shows that $\langle A, \prec\rangle \geq \nu^{*}$, as required.

We remark that Theorem 4.5 is best possible in the sense that there is a subset $A \subseteq{ }^{\nu} \kappa$ of cardinality $|A|=\left.\right|^{<\nu} \kappa \mid$ such that $\langle A, \prec\rangle$ has depth $\nu$. If $\nu=\mu^{+}$is a successor cardinal this is obvious (consider sequences of the form $f * O_{\nu}$, with $\left.f \in{ }^{\mu} \kappa\right)$. It is also clear if $\nu=\omega$ (choose $A$ well-ordered). Now suppose that $\nu>\omega$ is a limit cardinal and that $\left\langle\nu_{\xi}: \xi<\operatorname{cf}(\nu)\right\rangle$ is a sequence of infinite cardinals cofinal in $\nu$. Choose $f_{\xi} \in{ }^{\nu} \kappa(\xi<\operatorname{cf}(\nu))$ such that $f_{0} \prec f_{1} \prec \cdots$ and so that $\left(f_{\xi}, f_{\xi+1}\right)$ is a nonempty interval. There is a subset $A_{\xi} \subseteq\left(f_{\xi}, f_{\xi+1}\right)$ such that $\left|A_{\xi}\right|=\left|\nu^{\nu} \kappa\right|$ and $A_{\xi}$ has depth $\nu_{\xi}^{+}$. Then $A=\bigcup\left\{A_{\xi}: \xi<\operatorname{cf}(\nu)\right\}$ has depth $\nu$ and $|A|=|<\nu \kappa|$.

5. A Proof of Theorem 2.5. Before proving the theorem in general, we point out that for the special case when $\nu$ is regular and $\kappa$ is a strong limit cardinal of cofinality $\operatorname{cf}(\kappa)=\nu$ the result follows easily from Theorem 4.5. For, in this case, $\left.\right|^{<\nu} \kappa|=\kappa<|^{\nu} \kappa \mid$ and, so, if $\lambda \leq \kappa$ and $\left\{L_{\xi}: \xi<\lambda\right\}$ is any $\lambda$-decomposition of ${ }^{\nu} \kappa$, then $\left|L_{\xi}\right|>\kappa$ for some $\xi<\lambda$ and therefore has depth $\nu^{+}$by Theorem 4.5. This observation provides a simple proof of Theorem 2.7 in the case when $\nu$ is regular since, for any fixed $\lambda$, we can always choose a strong limit cardinal $\kappa>\lambda$ with cofinality $\nu$ (e.g. let $\kappa=\beth_{\lambda+\nu}$ ). The main difficulty in proving Theorem 2.7 is to establish Theorem 2.5 for the case when $\kappa>\nu$ and $\nu$ is a singular cardinal. As we have already mentioned, the proof given below for this case is much simpler than our original proof and uses an idea given to us by Z. Nagy.

Let $\nu \geq \omega, \kappa \geq 2, \lambda<\max \left\{\nu^{+}, \kappa\right\}$ and let $\left\langle L_{\xi}: \xi<\lambda\right\rangle$ be any $\lambda$-decomposition of ${ }^{\nu} \kappa$. We want to show that

$$
\operatorname{depth}\left(\left\langle L_{\xi}, \prec\right\rangle\right) \geq \nu^{+} \quad \text { for some } \xi<\lambda .
$$

Case 1. $\kappa \leq \nu^{+}$.

In this case $\lambda \leq \nu$ and, so, if (5.1) is false, then ${ }^{\nu} \kappa=\bigcup\left\{L_{\xi}: \xi<\lambda\right\}$ is of the first $(\operatorname{cf}(\nu))^{+}$-category by Theorem 2.6. But this contradicts Theorem 4.3 which implies that ${ }^{\nu} \kappa$ is of the second $(\operatorname{cf}(\nu))^{+}$-category. 
Case 2. $\kappa>\nu$.

In this case we can assume that $\lambda \geq \nu$. Suppose (5.1) is false. Then, by Corollary 2.3 , each $L_{\xi}$ can be partitioned into $\nu$ sets each having depth at most $\nu$, and so we may assume without loss of generality that

$$
\operatorname{depth}\left(\left\langle L_{\xi}, \prec\right\rangle\right) \leq \nu \text { for all } \xi<\lambda .
$$

For $\gamma<\nu, f \in{ }^{\nu} \kappa, X \subseteq{ }^{\nu} \kappa$, define $D_{\gamma}(f, X)=\{g \in X: \delta(f, g)=\gamma, f \prec g\}$, $\Gamma(f, X)=\left\{\gamma<\nu: D_{\gamma}(f, X) \neq \varnothing\right\}, B(X)=\{f \in X: \Gamma(f, X) \neq \nu\}$. Note that, if $f \in X \backslash B(X)$, then for each $\gamma<\nu$ there is $g_{\gamma} \in X$ such that $\delta\left(f, g_{\gamma}\right)=\gamma$ and $f \prec g_{\gamma}$. This implies that $g_{0} \succ g_{1} \succ \cdots \succ g_{\gamma} \succ \cdots$, i.e. $\langle X, \prec\rangle$ embeds $\nu^{*}$. It follows from (5.2) that $B\left(L_{\xi}\right)=L_{\xi}$ for all $\xi<\lambda$.

We may assume that the sets $L_{\xi}(\xi<\lambda)$ are pairwise disjoint so that, for any $g \in{ }^{\nu} \kappa$, there is a unique $\xi$ such that $g \in L_{\xi}=B\left(L_{\xi}\right)$. Since $\Gamma\left(g, L_{\xi}\right) \neq \nu$ we may define $\gamma(g)=\min \left(\nu \backslash \Gamma\left(g, L_{\xi}\right)\right)$. Then ${ }^{\nu} \kappa$ is the disjoint union of sets $L_{\xi \gamma}(\xi<\lambda, \gamma<\nu)$, where $L_{\xi \gamma}=\left\{g \in L_{\xi}: \gamma(g)=\gamma\right\}$. We shall obtain the desired contradiction by constructing, by transfinite induction, $f(\gamma) \in \kappa(\gamma<\nu)$ so that $f \notin L_{\xi \gamma}$ for all $\xi<\lambda$ and $\gamma<\nu$.

Let $\gamma<\nu$ and suppose we have already chosen $f(\rho) \in \kappa$ for $\rho<\gamma$. Let $f_{\gamma}=\langle f(\rho): \rho<\gamma\rangle \in{ }^{\gamma} \kappa$. Note that, if $\xi<\lambda$ and $g_{1}, g_{2} \in L_{\xi \gamma}$ are such that $g_{1} \uparrow \gamma=$ $g_{2} \uparrow \gamma$, then $g_{1}(\gamma)=g_{2}(\gamma)$. Otherwise, if, say, $g_{1}(\gamma)<g_{2}(\gamma)$, this contradicts the fact that $\gamma\left(g_{1}\right)=\gamma$. It follows that $E_{\xi \gamma}=\left\{g(\gamma): g \in L_{\xi \gamma}\right.$ and $g\left\lceil\gamma=f_{\gamma}\right\}$ contains at most one element. Since $\lambda<\kappa$ we may choose $f(\gamma)=\min \left(\kappa \backslash \bigcup_{\xi<\lambda} E_{\xi \gamma}\right)$. This defines $f \in{ }^{\nu} \kappa$. It is clear from the choice of $f(\gamma)$ that $f \notin L_{\xi \gamma}$ for any $\xi<\lambda$ and $\gamma<\nu$, and this is a contradiction since ${ }^{\nu} \kappa=\bigcup\left\{L_{\xi \gamma}: \xi<\lambda, \gamma<\nu\right\}$.

6. Proof of Theorem 2.8. Let $\mu>\omega$ be a strong limit cardinal and let $\langle P,<\rangle$ be a partially ordered set of dimension $\delta<\operatorname{cf}(\mu)$ and width $\mu$. We have to show that $P$ is a union of $\kappa=\left|{ }^{\delta} \operatorname{cf}(\mu)\right|$ sets $P_{\rho}(\rho<\kappa)$ each having width strictly less than $\mu$.

Let $<=\bigcap\left\{<_{\xi}: \xi<\delta\right\}$, where each $<_{\xi}$ is a total ordering of $P$. For $1 \leq \xi<\delta$ consider the partial order $<_{\xi}^{*}=<_{0} \cap>_{\xi}$ on $P$. If $X \subseteq P$ is totally ordered by $<_{\xi}^{*}$, then $X$ is an antichain of $\langle P,<\rangle$ and so $|X|<\mu$. Since $\left\langle P,<_{\xi}^{*}\right\rangle$ contains no chain of cardinality $\mu$, it follows that $\operatorname{depth}\left(\left\langle P,<_{\xi}^{*}\right\rangle\right) \leq \mu$ and $\left.\operatorname{depth}\left(\langle P,\rangle_{\xi}^{*}\right\rangle\right) \leq \mu$. By Theorem 2.4 (since any infinite cardinal is extendable), there is a $\operatorname{cf}(\mu)$-partition $\Delta_{\xi}^{0}=\left\{P_{\rho}^{\xi, 0}: \rho<\operatorname{cf}(\mu)\right\}$ of $P$ such that the depth of each $\left\langle P_{\rho}^{\xi, 0},<_{\xi}^{*}\right\rangle(\rho<\operatorname{cf}(\mu))$ is strictly less than $\mu$. Similarly, there is a $\operatorname{cf}(\mu)$-partition $\Delta_{\xi}^{1}=\left\{P_{\rho}^{\xi, 1}: \rho<\operatorname{cf}(\mu)\right\}$ of $P$ such that the depth of $\left\langle P_{\rho}^{\xi, 1},>_{\xi}^{*}\right\rangle(\rho<\operatorname{cf}(\mu))$ is also strictly less than $\mu$.

Now consider the common refinement $\Delta$ of all the partitions $\Delta_{\xi}^{0}, \Delta_{\xi}^{1}(\xi<\delta)$. Clearly, $|\Delta| \leq \kappa=\left|{ }^{\delta} \operatorname{cf}(\mu)\right|(\leq \mu)$. We claim that, for each $Q \in \Delta$, the width of $\langle Q,<\rangle, \mu(\langle Q,<\rangle)$, is strictly less than $\mu$.

To see this suppose, for contradiction, that $\langle Q,<\rangle$ has width $\geq \mu$. For each $\xi(1 \leq$ $\xi<\delta)$ there are $\rho(\xi, 0)$ and $\rho(\xi, 1)$ less than $\operatorname{cf}(\mu)$ such that $Q \subseteq P_{\rho(\xi, \varepsilon)}^{\xi, \varepsilon}(\varepsilon<2)$. Since $\delta<\operatorname{cf}(\mu)$, we can choose a cardinal $\zeta<\mu$ such that the depths of $\left\langle P_{\rho(\xi, 0)}^{\xi, 0},<_{\xi}^{*}\right\rangle$ and $\left\langle P_{\rho(\xi, 1)}^{\xi, 1},>_{\xi}^{*}\right\rangle$ are less than $\varsigma$ for all $\xi(1 \leq \xi<\delta)$. Since $\mu$ is a strong limit cardinal, there is a cardinal $\nu<\mu$ such that $\nu \rightarrow(\varsigma)_{\delta \cdot 2}^{2}$ (for example, $\nu=\left|{ }^{\varsigma+\delta+\omega} 2\right|^{+}$ will do-see [6, Corollary 17.5]). By our assumption on the width of $\langle Q,<\rangle$, there is 
an $A \subseteq Q$ such that $|A|=\nu$ and $A$ is an antichain of $\langle Q,<\rangle$. Let $A=\left\{a_{\alpha}: \alpha<\nu\right\}$ and consider the partition of $[\nu]^{2}$ into $\delta \cdot 2$ classes $K_{\varepsilon}^{\xi}(1 \leq \xi<\delta, \varepsilon=0$ or 1$)$ so that, for $\sigma<\tau<\dot{\nu}$, the pair $\{\sigma, \tau\}$ belongs to $K_{\varepsilon}^{\xi}$ if $\xi$ is the least ordinal such that the orders $<_{0}$ and $<_{\xi}$ disagree on the pair $\left\{a_{\sigma}, a_{\tau}\right\}$ (there is such a $\xi$ ), and $\varepsilon=0$ or 1 according as $a_{\sigma}<_{0} a_{\tau}$ or $a_{\tau}<_{0} a_{\sigma}$. From the partition relation $\nu \rightarrow(\zeta)_{\sigma \cdot 2}^{2}$ it follows that there are $\xi<\delta, \varepsilon<2$ and $B \subseteq \nu$ such that $|B|=\zeta$ and $[B]^{2} \subseteq K_{\varepsilon}^{\xi}$. It follows from this that if $A^{\prime}=\left\{a_{\sigma}: \sigma \in B\right\}$, then $>_{\xi}$ and $<_{0}$ agree on $A^{\prime}$ so that $\left\langle A^{\prime},<_{\xi}^{*}\right\rangle$ is a total order with the same order type as $\left\langle A^{\prime},<_{0}\right\rangle$. But this is $\geq \varsigma$ or $\geq \varsigma^{*}$ according as $\varepsilon=0$ or 1 . This implies that either $\left\langle P_{\rho(\xi, 0)}^{\xi, 0},<_{\xi}^{*}\right\rangle$ or $\left\langle P_{\rho(\xi, 1)}^{\xi, 1},>_{\xi}^{*}\right\rangle$ has depth greater than $\zeta$, which contradicts the choice of $\zeta$.

ACKNOWLEDGEMENT. The authors wish to express their appreciation to the referee and to $\mathrm{F}$. Galvin for several useful comments on the original version of this paper.

\section{REFERENCES}

1. J. Baumgartner and A. Hajnal, A proof (involving Martin's axiom) of a partition relation, Fund. Math. 78 (1973), 193-203.

2. R. Bonnet and M. Pouzet, Linear extensions of ordered sets, Ordered Sets (I. Rival, ed.), Reidel, Dordrecht, 1982, pp. 125-170.

3. R. P. Dilworth, A decomposition theorem for partially ordered sets, Ann. of Math. (2) 51 (1950), 161-166.

4. B. Dushnik and E. W. Miller, Partially ordered sets, Amer. J. Math. 63 (1941), 600-610.

5. P. Erdős and A. Hajnal, On decompositions of graphs, Acta. Math. Acad. Sci. Hungar. 18 (1967), 359-377.

6. P. E. Erdős, A. Hajnal, A. Máté and R. Rado, Combinatorial set theory, partition relations for cardinals, North-Holland, Amsterdam, 1984.

7. F. Galvin, On a partition theorem of Baumgartner and Hajnal, Colloq. Math. Soc. János Bolyai 10, North-Holland, Amsterdam, 1973, pp. 711-729.

8. T. Jech, Set theory, Academic Press, New York, 1978.

9. E. C. Milner and K. Prikry, The cofinality of a partially ordered set, Proc. London Math. Soc. (3) 46 (1983), 454-470.

10. E. C. Milner and R. Rado, The pigeon-hole principle for ordinal numbers, Proc. London Math. Soc. (3) 15 (1965), 750-768.

11. M. A. Perles, On Dilworth's theorem in the infinite case, Israel J. Math. 1 (1963), 108-109.

12. S. Todor ðević, Private communication.

13. __ Partition relations for partially ordered sets, Acta Math. (to appear).

Department of Mathematics \& Statistics, University of Calgary, Calgary, Alberta, CANADA T2N 1N4

Department of Mathematics, University of Minnesota, Minneapolis, MinNESOTA 55455 